DOI: $10.24850 /$ j-tyca-2018-06-08

Artículo

\title{
Modelos tarifarios de agua en México según criterios sociales
}

\section{Water pricing models in Mexico according to social criteria}

Hugo Briseño ${ }^{1}$

${ }^{1}$ Universidad Panamericana. Escuela de Ciencias Económicas y Empresariales. Prolongación Calzada Circunvalación Poniente 49, Zapopan, Jalisco, 45010, México.

hbriseno@up.edu.mx

Autor para correspondencia: Hugo Briseño, hbriseno@up.edu.mx

\section{Resumen}

El objetivo del presente artículo es proponer una tipología de modelos tarifarios basada en criterios sociales. En concreto, se plantean dos enfoques: diferenciación de usuarios y grupos vulnerables. Treinta y dos ciudades de México son agrupadas según su pertenencia a los enfoques antes mencionados con la finalidad de observar el comportamiento de sus indicadores de gestión promedio. Los resultados mostraron que el enfoque de diferenciación de usuarios hace más viable una tarifa más alta aunque la eficiencia del entre operador es más baja que en el de grupos vulnerables. En este último, la tarifa es menor y, por lo mismo, la eficiencia en la gestión es mayor. Asimismo, se observa que, con independencia del enfoque tarifario, las tarifas más altas van acompañadas de menor consumo. En conclusión, los incrementos en la tarifa de agua deben ir acompañados de una mayor eficiencia en la operación; ya que no es de utilidad que ingresen recursos financieros a una entidad, si éstos serán desperdiciados.

Palabras clave: modelos tarifarios, agua para uso urbano, criterios sociales, organismos operadores de agua.

\section{Abstract}


The purpose of this article was to propose a typology of pricing models based on social criteria. In particular, two approaches are proposed in this study: differentiation of users and vulnerable groups. Thirty-two cities of Mexico were grouped according to their abiding by the aforementioned approaches, to observe the behavior of their average management indicators. It turns out that the user differentiation approach makes a higher-rate model more feasible although the efficiency of the operating entity is lower than that of vulnerable groups. In the latter, the rate is lower, and therefore, management efficiency is greater. Likewise, it is observed that regardless of the pricing approach, higher rates are followed by lower consumption. It is concluded that increases in water rates should be accompanied by greater efficiency in the operation; because it is useless to enter financial resources into an entity if these will be wasted.

Keywords: pricing models, water for urban use, social criteria, water utilities.

Recibido: $12 / 04 / 2018$

Aceptado: 27/04/2018

\section{Introducción}

La tarifa del agua es un tema controversial, debido a que, en muchas ocasiones, es utilizado para lucrar políticamente. Sin embargo, dicha tarifa es un elemento fundamental en la gestión del agua, porque puede influir en el consumo del recurso hídrico y en la salud financiera de las entidades encargadas de llevarla a los hogares. Aunque se considera importante que la tarifa sea adecuada para cubrir los costos del servicio, es necesario que existan esquemas para asegurar un mínimo de agua a los hogares, con independencia de tener o no el recurso económico para adquirirla.

En la mayor parte de las ciudades de México, la estructura tarifaria del agua contempla algún beneficio para las clases sociales más vulnerables. El objetivo de este trabajo es identificar cuáles son los enfoques para determinar las tarifas según el criterio social y analizar cómo varía la gestión del agua en las ciudades según la perspectiva adaptada. En este trabajo se proponen dos enfoques principales: grupos 
vulnerables y diferenciación de usuarios. El primero, refiere a aquellas estructuras tarifarias que identifican usuarios con ciertas características que los hacen acreedores de recibir algunos beneficios en cuanto al pago, principalmente descuentos. El segundo, divide a los usuarios según su nivel socioeconómico, con la finalidad de asignar tarifas diferenciadas.

En los siguientes apartados se realizará una breve revisión de la literatura sobre los modelos tarifarios; se explicará la metodología utilizada para la determinación de los enfoques, así como la gestión de las entidades que los adoptan; se mostrarán los resultados, y se expondrán una serie de conclusiones acerca de las ventajas y desventajas de cada enfoque.

\section{Revisión sobre modelos tarifarios}

A diferencia de otros productos y servicios, cuyo fin principal del precio es maximizar la utilidad de una empresa o un negocio; la tarifa de agua persigue varios objetivos: cuidar el medio ambiente, eficiencia en el uso del recurso, equidad entre diferentes usuarios y conservación, entre otros (Molinos-Senante \& Donoso, 2016; Dinar, Pochat \& Albiac-Murillo, 2015; Grafton, Chu \& Kompas, 2015; Rogers, de Silva \& Bhatia, 2002). Cabe señalar, que para que sean sostenibles los sistemas de distribución de agua se requieren grandes inversiones en el mantenimiento y reposición constante de la infraestructura (Enouy, Rehan, Brisley \& Unger, 2015), lo que hace indispensable la disponibilidad de recursos financieros para tal fin.

Aunado a lo ya mencionado es fundamental que la tarifa desincentive los altos consumos y, sobretodo, que castigue los excesos en el gasto de agua. Molinos-Senante y Donoso (2016) señalan la importancia de que la tarifa del agua internalice los desperdicios de la misma. En este sentido, la tarifa es una herramienta fundamental para regular el gasto de este recurso tan esencial para la vida, y por lo tanto, la vuelve un elemento que recibe la expectación de actores, tanto políticos como mediáticos. Sin embargo, no es nada sencillo llegar a acuerdos sobre el nivel de la tarifa ni su estructura. Existen costos de oportunidad entre la equidad, la eficiencia económica y la recuperación de costos (Nauges \& Whittington, 2017). Por ejemplo, una tarifa alta, sin excepciones podría 
abonar a la sustentabilidad financiera de los organismos operadores, no así a la equidad entre los usuarios del sistema.

Definir el nivel del precio del agua en las ciudades es un tema complicado, que conlleva costos en la popularidad de los tomadores de decisiones. Herrera (2017) señala la importancia que tiene la política en la toma de decisiones referente al agua; a través de estudios de casos en diferentes localidades de México, señala que el recurso muchas veces es utilizado para ganar adeptos electorales. Es decir, la tarifa está altamente influenciada por criterios políticos, que en la mayoría de las ocasiones, están alejados a lo técnicamente conveniente. Por tal razón, las tarifas no siempre son suficientes para cubrir los costos operativos en los que incurren las entidades encargadas de suministrar el agua a las ciudades. Esto, trae como consecuencia que los organismos operadores no tengan los recursos para llevar a cabo obras de infraestructura para captar y distribuir el agua, por lo que se entra en un círculo perverso en el que se hace cada vez más costoso llevar el servicio a la población, misma que no paga lo suficiente por el mismo. Spiller y Savedoff (1999), bajo la perspectiva del equilibrio de bajo nivel, señalan que la baja eficiencia en la operación de los organismos va acompañada de baja calidad en el servicio y baja voluntad de la población para pagarlo.

Existen diferentes estructuras tarifarias que persiguen diversos objetivos. Algunas de ellas son las siguientes (Molinos-Senante \& Donoso, 2016; Pinto \& Marques, 2015), cargo variable por volumen uniforme, tarifa de incremento por bloque, tarifa decreciente por bloque, tarifa de temporada, tarifa de tiempo de uso y tarifa espacial. El grueso de estas estructuras se enfoca, sobre todo, en desincentivar o incentivar el uso de agua según las características y disponibilidad del recurso en un lugar y tiempo específico. Por ejemplo, la tarifa de incremento por bloque busca alentar a los usuarios a que se mantengan en los primeros bloques de consumo; mientras que la tarifa por bloque decreciente incentiva el consumo, atendiendo a las economías de escala que se generan al prorratear los costos fijos entre más metros cúbicos, aunque no se promueve la conservación ni una percepción de escasez entre la población atendida.

Pinto \& Marques (2015), citando a Johnson (1971), señalan que la estructura tarifaria apropiada para cada localidad depende de sus propias circunstancias y los objetivos que se persigan; y rescatan las diferentes políticas descritas en la literatura, mismas que se pueden observar en la Tabla 1. 
Tabla 1. Políticas tarifarias según la literatura.

\begin{tabular}{|c|c|c|c|}
\hline Tarifa & Definición & $\begin{array}{l}\text { aracterísticas } \\
\text { e diseño }\end{array}$ & $\begin{array}{l}\text { Características } \\
\text { Teóricas }\end{array}$ \\
\hline flana & \begin{tabular}{|lr} 
Cada r usuario \\
paga una ruota \\
que r r no \\
depende & del \\
volumen & de \\
agua utilizada &
\end{tabular} & $\begin{array}{l}\text { 1) El precio } \\
\text { que se } \\
\text { cargará }\end{array}$ & $\begin{array}{l}\text { Simpleza } \\
\text { administrativa, no } \\
\text { requiere metro, } \\
\text { baja eficiencia } \\
\text { económica, baja } \\
\text { equidad }\end{array}$ \\
\hline $\begin{array}{l}\text { Bloque único } \\
\text { uniforme } \\
\text { (Uniform, } \\
\text { single block - } \\
\text { UT) }\end{array}$ & $\begin{array}{lr}\text { Se paga } & \text { una } \\
\text { tarifa } & \text { por } \\
\text { metro } & \text { cúbico } \\
\text { que no cambia } \\
\text { según } & \text { el } \\
\text { volumen } & \end{array}$ & $\begin{array}{l}\text { 1) El precio } \\
\text { por metro } \\
\text { cúbico. }\end{array}$ & $\begin{array}{l}\text { Es } \\
\text { administrativament } \\
\text { e simple, alienta la } \\
\text { conservación, y } \\
\text { manda una señal de } \\
\text { escasez }\end{array}$ \\
\hline $\begin{array}{l}\text { Tarifa de } \\
\text { bloque } \\
\text { creciente } \\
\text { (Increasing } \\
\text { block - IBT) }\end{array}$ & $\begin{array}{lr}\text { El cargo } & \text { se } \\
\text { incrementa } & \\
\text { según } & \text { el } \\
\text { volumen } & \\
\text { consumido } & \text { de } \\
\text { un bloque } & \text { a } \\
\text { otro } & \end{array}$ & $\begin{array}{l}\text { 1) Número de } \\
\text { bloques } \\
\text { 2) Volumen } \\
\text { de agua } \\
\text { asociada a } \\
\text { cada } \\
\text { bloque } \\
\text { 3) Precio que } \\
\text { será } \\
\text { cargado } \\
\text { por cada } \\
\text { bloque }\end{array}$ & $\begin{array}{l}\text { Alienta la } \\
\text { conservación, } \\
\text { manda una señal de } \\
\text { escasez de agua, } \\
\text { promueve equidad }\end{array}$ \\
\hline $\begin{array}{l}\text { Tarifa de } \\
\text { bloque } \\
\text { decreciente } \\
\text { (Decreasing } \\
\text { block - DBT) }\end{array}$ & $\begin{array}{lr}\text { El } & \text { cargo } \\
\text { disminuye } & \\
\text { según } & \text { el } \\
\text { volumen } & \\
\text { consumido } & \text { de } \\
\text { un bloque } & \text { a } \\
\text { otro } & \end{array}$ & $\begin{array}{l}\text { 1) Número de } \\
\text { bloques. } \\
\text { 2) Volumen } \\
\text { de agua } \\
\text { asociada a } \\
\text { cada } \\
\text { bloque. } \\
\text { 3) Precio que } \\
\text { será } \\
\text { cargado } \\
\text { por cada } \\
\text { bloque }\end{array}$ & $\begin{array}{l}\text { Refleja los costos, } \\
\text { no alienta la } \\
\text { conservación. Aplica } \\
\text { a áreas donde hay } \\
\text { una gran cantidad } \\
\text { de agua para } \\
\text { vender }\end{array}$ \\
\hline & $\begin{array}{|lr|}\text { El } & \text { precio } \\
\text { unitario } & \text { del } \\
\end{array}$ & $\begin{array}{l}\text { 1) Número de } \\
\text { bloques }\end{array}$ & $\begin{array}{l}\text { Se } \\
\text { simple, }\end{array}$ \\
\hline
\end{tabular}




\begin{tabular}{|c|c|c|c|}
\hline $\begin{array}{l}\text { escala móvil } \\
\text { (Increasing } \\
\text { rate - IRT) }\end{array}$ & $\begin{array}{l}\text { total de agua } \\
\text { utilizada se } \\
\text { incrementa } \\
\text { como aumenta } \\
\text { la cantidad } \\
\text { consumida }\end{array}$ & $\begin{array}{l}\text { 2) Volumen } \\
\text { de agua } \\
\text { asociado a } \\
\text { cada } \\
\text { bloque } \\
\text { 3) Precio que } \\
\text { será } \\
\text { cargado } \\
\text { según el } \\
\text { último } \\
\text { bloque } \\
\text { consumido }\end{array}$ & $\begin{array}{l}\text { conservación, } \\
\text { manda la señal de } \\
\text { escasez y promueve } \\
\text { la equidad }\end{array}$ \\
\hline \multicolumn{4}{|l|}{$\begin{array}{l}\text { Ajustes } \\
\text { Tarifarios }\end{array}$} \\
\hline $\begin{array}{ll}\text { Según } & \text { los } \\
\text { usuarios } & \end{array}$ & $\begin{array}{l}\text { Diferenciación } \\
\text { de las tarifas } \\
\text { en base a } \\
\text { características } \\
\text { de los usuarios }\end{array}$ & $\begin{array}{l}\text { Se diseña la } \\
\text { tarifa (cargo fijo } \\
\text { y variable) según } \\
\text { el tipo de } \\
\text { consumidor }\end{array}$ & $\begin{array}{l}\text { Puede introducir } \\
\text { principios de } \\
\text { eficiencia y equidad. } \\
\text { Es } \\
\text { administrativament } \\
\text { e complejo }\end{array}$ \\
\hline $\begin{array}{l}\text { Según } \\
\text { estacionalida } \\
\text { d }\end{array}$ & $\begin{array}{lr}\text { Tarifas } & \text { más } \\
\text { altas durante } \\
\text { periodos de } \\
\text { mayor } \\
\text { demanda }\end{array}$ & $\begin{array}{ll}\text { Cambios } & \text { de } \\
\text { precio según la } \\
\text { demanda }\end{array}$ & $\begin{array}{l}\text { Alienta } \\
\text { conservación }\end{array}$ \\
\hline $\begin{array}{l}\text { Según hora } \\
\text { de uso }\end{array}$ & $\begin{array}{l}\text { Tarifa más alta } \\
\text { durante horas } \\
\text { de mayor } \\
\text { consumo }\end{array}$ & $\begin{array}{ll}\text { Cambios } & \text { de } \\
\text { precio según la } \\
\text { demanda }\end{array}$ & $\begin{array}{ll}\text { Alienta la } \\
\text { conservación. } \\
\text { Requiere sistemas } \\
\text { de } & \\
\text { avanzados medición } & \\
\end{array}$ \\
\hline $\begin{array}{l}\text { Según } \\
\text { lugar }\end{array}$ & $\begin{array}{l}\text { Usuarios pagan } \\
\text { el costo de } \\
\text { suministro } \\
\text { hacia su } \\
\text { establecimient } \\
0\end{array}$ & $\begin{array}{l}\text { El diseño de } \\
\text { tarifa se adapta } \\
\text { según el costo } \\
\text { que implique } \\
\text { llevarla a un } \\
\text { establecimiento } \\
\text { en específico }\end{array}$ & $\begin{array}{lr}\text { Principios } & \text { de } \\
\text { asignación y } & \text { costo } \\
\text { eficientes, } & \text { baja } \\
\text { equidad, } & \text { baja } \\
\text { asequibilidad } & \end{array}$ \\
\hline
\end{tabular}

Fuente: Pinto \& Marques (2015) traducido por el autor y con algunas adaptaciones 
En México, por lo general, las tarifas del agua para uso urbano no son suficientes para cubrir los costos del servicio, por los que los gobiernos deben salvar a los organismos operadores a través de transferencias de recursos económicos. Además, existe el subsidio cruzado, que consiste en que los ingresos generados por la cobranza a usuarios comerciales e industriales, a quienes se les recauda una tarifa más alta, sirvan para compensar el precio abajo de costo que se les cobra a los usuarios domésticos. Si se abusa de este recurso puede haber afectaciones importantes en dos sentidos: desincentivar la actividad económica, por un excesivo precio del agua a usuarios industriales o comerciales; $y$, enviar una señal equivocada a los usuarios domésticos, en el sentido de que el recurso no es escaso o no cuesta proveerlo. Cuando sucede esto último se corre el riesgo de que el usuario tienda a utilizar más agua que la que realmente necesita.

Como se pudo observar en la Tabla 1, existen esquemas tarifarios según diferentes objetivos planteados (Pinto \& Marques, 2015):

- Tarifa plana: cada usuario paga una cuota fija, existe facilidad administrativa, pero no hay una señal de escasez ni de conservación.

- Bloque único uniforme: se paga por metro cúbico, es administrativamente simple y ya hay una señal de escasez.

- Tarifa de bloque creciente: en cada bloque se carga una tarifa más alta, esto desincentiva los altos consumos y promueve la equidad.

- Tarifa de bloque decreciente: la tarifa refleja los costos, pero no alienta a la conservación.

- Tarifa creciente: se considera relativamente simple, envía una señal de escasez y fomenta la equidad.

Aunque los autores Pinto y Marques (2015) rescatan que las tarifas pueden variar en diseño según el tipo de usuario, no profundizan en este tema que pudiera ser decisivo en el nivel y consecuencias de la tarifa. En este artículo se pondrá atención en aquellos enfoques tarifarios, compatibles con las estructuras antes mencionadas, que buscan asegurar el suministro de las personas menos favorecidas económicamente. En México, existen dos tipos de orientaciones tarifarias; en cuanto al aspecto social: una que divide a los usuarios de la ciudad según la zona geográfica donde viven, con la finalidad de cobrarle más, a quienes supuestamente más tienen y menos, a quienes, en apariencia, tienen poco. Otra que mantiene una misma estructura tarifaria en cuanto a bloques y montos para la mayoría de la población, pero hace excepciones reduciendo la cantidad a pagar en ciertos rangos de consumo para aquellas personas con cierta vulnerabilidad, como son 
los pobres y ancianos. En las siguientes páginas se explicará cómo se seleccionaron algunas ciudades de México para categorizarlas en cada uno de los enfoques sociales, para revisar los indicadores de gestión de sus entes encargados de suministrar y administrar el recurso en cuestión.

\section{Metodología}

Se generó una base de datos de 32 ciudades (Tabla 2) con información extraída del Informe Situación del Subsector de Agua Potable, Alcantarillado y Saneamiento, Edición 2014 de la Comisión Nacional del Agua (CONAGUA). De cada ciudad se obtuvieron los siguientes datos: tarifas domésticas de 2008 a 2013; tarifas comercial e industrial; eficiencia física, eficiencia comercial y eficiencia global, dotación, ingreso, y costo por metro cúbico. Con esta información se construyeron los siguientes indicadores: incremento porcentual en tarifas domésticas de 2008 a 2013; nivel de subsidio cruzado (promedio tarifa industrial y comercial entre tarifa doméstica); dotación real (dotación por eficiencia física); y utilidad por metro cúbico (diferencia entre ingreso y costo por metro cúbico). Cabe señalar que, por subsidio cruzado se entiende que los usuarios comerciales e industriales, a través de tarifas sustancialmente más altas, compensan la tarifa más baja que pagan los usuarios domésticos.

Tabla 2. Base de datos

\begin{tabular}{|c|c|c|c|c|c|c|c|c|c|c|c|c|c|}
\hline & Ciudad & $\begin{array}{c}\text { Tarifa } \\
\text { doméstica }\end{array}$ & $\begin{array}{c}\text { Incremento } \\
\text { tarifa } \\
\text { doméstica } \\
2008- \\
2013\end{array}$ & $\begin{array}{c}\text { Promedio } \\
\text { tarifas } \\
\text { comercial } \\
e \\
\text { industrial }\end{array}$ & $\begin{array}{c}\text { Cobertura } \\
(\%)\end{array}$ & $\begin{array}{l}\text { Eficiencia } \\
\text { física } \\
(\%)\end{array}$ & $\begin{array}{c}\text { Eficiencia } \\
\text { comercial } \\
(\%)\end{array}$ & $\begin{array}{l}\text { Eficiencia } \\
\text { global } \\
(\%)\end{array}$ & $\begin{array}{c}\text { Dotación } \\
\text { (Its/hab/día) }\end{array}$ & $\begin{array}{c}\text { Dotación } \\
\text { real } \\
\text { (Its/hab/día) }\end{array}$ & $\begin{array}{l}\text { Utilidad } \\
\text { por } \\
\text { metro } \\
\text { cúbico }\end{array}$ & $\begin{array}{l}\text { Nivel de } \\
\text { subsidio } \\
\text { cruzado }\end{array}$ & $\begin{array}{l}\text { Modelo } \\
\text { Tarifario } \\
\text { (E. Dif. = } \\
\text { 1) }\end{array}$ \\
\hline 1 & Acapulco & 11 & $75 \%$ & 27.20 & 91.00 & 41.97 & 61.90 & 25.98 & 394.59 & 165.61 & -1.95 & 2.47 & 0 \\
\hline 2 & Aguascalientes & 20 & $40 \%$ & 55.79 & 99.27 & 63.00 & 93.00 & 59.00 & 299.76 & 188.85 & 0.06 & 2.79 & 1 \\
\hline 3 & Atizapán & 15.8 & $44 \%$ & 54.40 & 99.00 & & 53.85 & & 238.78 & & -5.53 & 3.44 & 1 \\
\hline 4 & Campeche & 1.9 & $19 \%$ & 4.50 & 94.00 & 41.10 & 70.36 & 28.91 & 496.03 & 203.87 & -1.08 & 2.37 & 1 \\
\hline 5 & Cancún & 11 & $90 \%$ & 10.75 & & & & & & & & 0.98 & 0 \\
\hline 6 & Chetumal & 11 & $90 \%$ & 39.90 & 98.20 & 30.18 & 55.87 & 16.86 & 415.02 & 125.25 & -2.51 & 3.63 & 0 \\
\hline 7 & Colima & 4.5 & $25 \%$ & 6.30 & & & & & & & -1.59 & 1.40 & 1 \\
\hline 8 & Culiacán & 5.8 & $57 \%$ & 40.48 & 99.45 & 64.95 & 90.32 & 58.66 & 268.45 & 174.36 & -1.38 & 6.98 & 0 \\
\hline 9 & Delicias & 5.4 & $10 \%$ & 8.05 & & 44.85 & 76.58 & 34.34 & & & -2.39 & 1.49 & 0 \\
\hline 10 & Distrito Federal & 16.8 & $243 \%$ & 26.90 & 97.55 & 53.68 & 73.95 & 39.70 & 302.93 & 162.61 & -3.48 & 1.60 & 1 \\
\hline 11 & Ensenada & 15.3 & $40 \%$ & 48.20 & 99.40 & 82.40 & 89.79 & 73.99 & 199.27 & 164.20 & -5.94 & 3.15 & 0 \\
\hline 12 & Gómez Palacio & 7.7 & $24 \%$ & 24.80 & & 55.34 & 87.47 & 48.41 & & & -1.89 & 3.22 & 1 \\
\hline 13 & Guadalajara & 8.6 & $65 \%$ & 16.75 & 98.00 & 71.63 & 84.63 & 60.62 & 208.55 & 149.38 & & 1.95 & 0 \\
\hline 14 & Hermosillo & 4.7 & $31 \%$ & 20.20 & 94.25 & 57.12 & 73.87 & 42.19 & 363.39 & 207.57 & -4.32 & 4.30 & 0 \\
\hline 15 & Juárez & 8.8 & $193 \%$ & 5.05 & 90.00 & 78.93 & 58.85 & 46.45 & 356.02 & 281.01 & & 0.57 & 0 \\
\hline 16 & La Paz & 9 & $55 \%$ & 18.55 & 98.07 & & 39.28 & & 195.34 & & & 2.06 & 0 \\
\hline
\end{tabular}




\begin{tabular}{|c|c|c|c|c|c|c|c|c|c|c|c|c|c|}
\hline 17 & León & 18.3 & $68 \%$ & 32.05 & 98.93 & 66.78 & 100.00 & 68.32 & 175.25 & 117.03 & 0.28 & 1.75 & 0 \\
\hline 18 & Mérida & 3.9 & $15 \%$ & 5.70 & 98.00 & 44.13 & 91.92 & 40.57 & 290.65 & 128.26 & -1.98 & 1.46 & 1 \\
\hline 19 & Mexicali & 4.9 & $40 \%$ & 31.90 & 99.70 & 84.19 & 89.42 & 75.28 & 300.49 & 252.98 & -4.69 & 6.51 & 0 \\
\hline 20 & Monterrey & 10.8 & $61 \%$ & 16.00 & 99.62 & 75.33 & 88.49 & 66.66 & 248.75 & 187.38 & & 1.48 & 1 \\
\hline 21 & Morelia & 19.7 & $66 \%$ & 47.63 & 90.00 & 39.07 & 40.88 & 15.97 & 407.07 & 159.04 & -3.61 & 2.42 & 1 \\
\hline 22 & Naucalpan & 18.4 & $79 \%$ & 31.10 & 98.00 & 46.23 & 63.09 & 29.17 & 346.30 & 160.09 & & 1.69 & 1 \\
\hline 23 & Oaxaca & 6.4 & $300 \%$ & 14.50 & & 36.34 & 49.92 & 18.14 & & & -9.17 & 2.27 & 1 \\
\hline 24 & Puebla & 10.9 & $31 \%$ & 13.50 & 95.85 & 62.15 & 59.24 & 36.82 & 170.99 & 106.27 & 0.66 & 1.24 & 0 \\
\hline 25 & $\begin{array}{c}\text { San Juan del } \\
\text { Río }\end{array}$ & 5.7 & $16 \%$ & 16.55 & 97.50 & 60.93 & 70.17 & 42.75 & 248.50 & 151.41 & -3.67 & 2.90 & 0 \\
\hline 26 & San Luis Potosí & 8.6 & $43 \%$ & 16.30 & & & & & & & & 1.90 & 1 \\
\hline 27 & Tijuana & 19.2 & $43 \%$ & 48.10 & 98.70 & 82.02 & 73.86 & 60.58 & 176.81 & 145.02 & -6.72 & 2.51 & 0 \\
\hline 28 & Tlaxcala & 5.3 & $51 \%$ & 27.00 & & & & & & & & 5.09 & 0 \\
\hline 29 & Toluca & 10.4 & $41 \%$ & 19.30 & 93.00 & & 40.40 & & 186.47 & & & 1.86 & 1 \\
\hline 30 & Torreón & 8 & $33 \%$ & 16.05 & 99.00 & 48.01 & 87.71 & 42.11 & 280.12 & 134.49 & -1.55 & 2.01 & 0 \\
\hline 31 & Tula de Allende & 4.9 & $53 \%$ & 13.05 & 80.00 & 51.42 & 86.74 & 44.60 & 331.64 & 170.53 & -0.8 & 2.66 & 1 \\
\hline 32 & Xalapa & 10.5 & $44 \%$ & 12.75 & 94.42 & 39.62 & 92.10 & 36.49 & 317.32 & 125.72 & & 1.21 & 1 \\
\hline
\end{tabular}

Fuente: elaboración propia con información de CONAGUA (2014)

En la Tabla 3 se pueden observar las tarifas para usuarios domésticos para un consumo de $30 \mathrm{~m}^{3}$ por mes. Se encontró un amplio rango de valores, desde ciudades como Campeche, con un cobro de 1.9 pesos por $\mathrm{m}^{3}$, hasta Aguascalientes con un cobro de 20 pesos. Esto refiere el gran mosaico de circunstancias que rodean la gestión del agua en cada una de las ciudades analizadas.

Tabla 3. Tarifas promedio por ciudad

\begin{tabular}{|l|l|l|l|}
\hline \multicolumn{4}{|c}{ Pesos por metro cúbico en consumos de $30 \mathbf{~ m}^{\mathbf{3}}$ / mes } \\
\hline $\begin{array}{l}\text { Aguascalientes } \\
(20)\end{array}$ & Acapulco (11) & Juárez (8.8) & Delicias (5.4) \\
\hline Morelia (19.7) & Cancún (11) & $\begin{array}{l}\text { San Luis Potosí } \\
(8.6)\end{array}$ & Tlaxcala (5.3) \\
\hline Tijuana (19.2) & Chetumal (11) & $\begin{array}{l}\text { Guadalajara } \\
(8.6)\end{array}$ & $\begin{array}{l}\text { Tula de Allende } \\
(4.9)\end{array}$ \\
\hline $\begin{array}{l}\text { Naucalpan } \\
(18.4)\end{array}$ & Puebla (10.9) & Torreón (8) & Mexicali (4.9) \\
\hline $\begin{array}{l}\text { León (18.3) } \\
\text { Distrito Federal } \\
(16.8)\end{array}$ & $\begin{array}{l}\text { Monterrey } \\
(10.8)\end{array}$ & $\begin{array}{l}\text { Gómez Palacio } \\
(7.7)\end{array}$ & Hermosillo (4.7) \\
\hline Atizapán (15.8) & Toluca (10.4) & Oaxaca (6.4) & Colima (4.5) \\
\hline $\begin{array}{l}\text { Ensenada } \\
(15.3)\end{array}$ & La Paz (9) & $\begin{array}{l}\text { San Jún (5.8) } \\
\text { Río (5.7) }\end{array}$ & Mérida (3.9) \\
\hline
\end{tabular}

Fuente: CONAGUA (2014) 
De los resolutivos tarifarios se obtuvo el modelo tarifario, con base en el siguiente criterio: si la tarifa es diferente para cada nivel socioeconómico o zona entonces se le llama enfoque de diferenciación de usuarios; en cambio, si la tarifa es similar para la mayor parte de la población, pero hay excepciones para ciertos grupos, entonces le denomina como enfoque de grupos vulnerables. Es decir, en el primer enfoque, la población se divide en grupos según sus capacidades económicas, y en el segundo, hay una misma estructura para la gran mayoría de usuarios, pero concediendo facilidades a ciertos grupos. En la Tabla 4 se observa la información que fue extraída del informe de la CONAGUA (2014), así como la construida con estos datos.

Tabla 4. Información extraída y construida

\begin{tabular}{|c|c|}
\hline $\begin{array}{c}\text { Información extraída del } \\
\text { Informe de la Conagua } \\
(2014)\end{array}$ & Datos construidos \\
\hline $\begin{array}{l}\text { Tarifas domésticas } 2008 \text { - } \\
2013 \\
\text { Tarifa comercial e industrial } \\
\text { Eficiencia física, comercial y } \\
\text { global } \\
\text { Dotación } \\
\text { Ingreso y costo por } \mathrm{m}^{3}\end{array}$ & $\begin{array}{l}\text { Incremento porcentual tarifas } \\
\text { domésticas } \\
\text { Nivel de subsidio cruzado (promedio } \\
\text { tarifa industrial y comercial entre tarifa } \\
\text { doméstica) } \\
\text { Dotación real (Dotación por Eficiencia } \\
\text { Física) } \\
\begin{array}{l}\text { Utilidad por m3 (Diferencia entre } \\
\text { Ingreso y costo por } \mathrm{m}^{3} \text { ) } \\
\text { Modelo tarifario (resolutivos) }\end{array}\end{array}$ \\
\hline
\end{tabular}

Fuente: elaboración propia

En la Tabla 5 se exponen cuáles son los principales criterios para determinar las tarifas en cada una de las ciudades seleccionadas. Como ya se había señalado, destacan dos principales esquemas generales: dividir la población entre usuarios con diferentes niveles socioeconómicos; o esquemas en los cuales, hay unos rangos tarifarios generales según el consumo, pero hay grupos vulnerables que tienen una tarifa subsidiada. Se encontraron casos en los que sólo existe una 
tarifa especial para personas que viven en residencia popular, hasta casos que tienen desde 4 hasta 8 escalones para diferenciar a los usuarios.

Tabla 5. Criterio social para determinar la tarifa de agua

\begin{tabular}{|c|c|}
\hline $\begin{array}{c}\text { Ciudad / } \\
\text { Organismo } \\
\text { operador de } \\
\text { agua } \\
\end{array}$ & Criterio social \\
\hline Acapulco & Tarifa especial para usuarios en residencia popular \\
\hline Aguascalientes & $\begin{array}{l}\text { Existe el doméstico urbano y el doméstico rural. El } \\
\text { primero tiene dos divisiones y el segundo tres }\end{array}$ \\
\hline Atizapán & $\begin{array}{l}\text { Popular, residencial y residencial alto. Tiene otra para } \\
\text { hospitales, asilos, guarderías, asociaciones civiles y } \\
\text { patronatos sin fines de lucro }\end{array}$ \\
\hline Campeche & $\begin{array}{l}\text { Diferenciación solo en cuota fija. Popular, } \\
\text { fraccionamientos, barrios y residencial }\end{array}$ \\
\hline $\begin{array}{l}\text { Cancún } \\
\text { Chetumal }\end{array}$ & $\begin{array}{l}\text { Existe una tabla con rangos de consumo base y otra } \\
\text { tabla con los subsidios en relación a la primera tabla } \\
\text { No hay diferenciación ni enfoque a grupos vulnerables. } \\
\text { El subsidio es general }\end{array}$ \\
\hline Colima & $\begin{array}{l}\text { Popular, medio, residencial } 1 \text { y residencial } 2 \text {. En la ley } \\
\text { se especifica qué colonias son las que están inscritas } \\
\text { en cada régimen }\end{array}$ \\
\hline Distrito Federal & $\begin{array}{l}\text { Popular, baja, media y alta. Se tipifican según el índice } \\
\text { de desarrollo de cada manzana que se compone de } \\
\text { tres variables (marginación, ingresos y valores } \\
\text { catastrales promedio) }\end{array}$ \\
\hline Ensenada & $\begin{array}{l}\text { Subsidio para pensionados, jubilados, discapacitados, } \\
\text { adultos mayores y viudos. Es del } 100 \% \text { para } \\
\text { consumos menores a } 25 \mathrm{~m}^{3} \text {; de } 26 \text { a } 40 \text { se subsidia el } \\
50 \% \text {; y más de } 41 \text { no aplica el subsidio }\end{array}$ \\
\hline Culiacán & $\begin{array}{l}\text { Existe una prestación que tienen las personas } \\
\text { pensionadas, las cuales solo deben de reunir ciertos } \\
\text { requisitos tales como el tarjetón de pensión, el último } \\
\text { talón de cheque, ser categoría doméstica y no } \\
\text { consumir más de } 25 \mathrm{~m}^{3} \text { durante tres meses } \\
\text { consecutivos }\end{array}$ \\
\hline Delicias & $\begin{array}{l}\text { Subsidio a pensionados con incapacidad de trabajar, } \\
\text { jubilados, damnificados, pobreza extrema, } \\
\text { discapacitados y mayores a } 60 \text { años }\end{array}$ \\
\hline
\end{tabular}




\begin{tabular}{|c|c|}
\hline $\begin{array}{l}\text { Gómez } \\
\text { Palacios } \\
\end{array}$ & $\begin{array}{l}\text { Doméstica subsistencia, rural y urbano. No están } \\
\text { claros los criterios para ser tipificado }\end{array}$ \\
\hline Guadalajara & $\begin{array}{l}\text { Tarifa zona de pobreza (menos de } 0.68 \text { salarios } \\
\text { mínimos al mes, sin alberca, con medidor, uso } \\
\text { habitacional y consumo de menos de } 21 \mathrm{~m}^{3} \text { al mes). } \\
\text { Tarifa viudos, jubilados, pensionados, discapacitados o } \\
\text { más de } 60 \text { años }\end{array}$ \\
\hline Hermosillo & $\begin{array}{l}\text { Tarifa social a no más del } 20 \% \text { de los usuarios } \\
\text { domésticos que cumplan con ciertas características en } \\
\text { el predio señaladas en la ley y sea pensionado o } \\
\text { jubilado, discapacitado, viuda o adulto mayor }\end{array}$ \\
\hline Juárez & $\begin{array}{l}50 \% \text { a pensionados, jubilados, mayores a } 60 \text { años. } \\
\text { Además, podrán recibir subsidio los damnificados y los } \\
\text { usuarios en pobreza extrema }\end{array}$ \\
\hline La Paz & No hay diferenciación ni subsidio \\
\hline León & No hay subsidios \\
\hline Morelia & $\begin{array}{l}\text { Divide a la población en cuatro zonas de servicio con } \\
\text { tarifa diferenciada: popular, popular A, media y } \\
\text { residencial. Existe también un subsidio del } 50 \% \text { para } \\
\text { adultos mayores y discapacitados, siempre y cuando } \\
\text { acrediten su situación y su casa tenga medidor }\end{array}$ \\
\hline Mérida & $\begin{array}{l}\text { Divide a los usuarios domésticas O USUSARIOS } \\
\text { DOMÉSTICOS en zona } 1,2 \text { y } 3 \text {. Pero a partir de } 21 \mathrm{~m}^{3} \\
\text { la estructura tarifaria es la misma. En la página de } \\
\text { internet aparecen las colonias por zonas, pero no se } \\
\text { explica el criterio utilizado }\end{array}$ \\
\hline Mexicali & $\begin{array}{l}\text { Exención del pago del agua para personas mayores de } \\
60 \text { años de escasos recursos, viudas mayores de } 50 \\
\text { años de escasos recursos, discapacitados, jubilados } \\
\text { y/o pensionados que no obtengan ingresos mayores a } \\
\text { cinco veces el salario mínimo general. Exención del } \\
\text { pago correspondiente por servicio de consumo de } \\
\text { agua a instituciones de asistencia social }\end{array}$ \\
\hline Monterrey & $\begin{array}{l}\text { Hay una tarifa especial para personas mayores de } 70 \\
\text { años, jubilados, pensionados, viudas y discapacitados. } \\
\text { Además, hay diferenciación entre las zonas foráneas } \\
\text { según su índice de marginalidad }\end{array}$ \\
\hline Naucalpan & $\begin{array}{l}\text { Popular, popular con tandeo, residencial medio y } \\
\text { residencial alto }\end{array}$ \\
\hline Oax & \\
\hline
\end{tabular}


Tecnología y

Ciencias $₫$ Agua

\begin{tabular}{|c|c|}
\hline Puebla & $\begin{array}{l}\text { Jubilados, pensionados, viudas, discapacitados y } \\
\text { adultos mayores el apoyo del } 50 \% \text { del pago de } \\
\text { servicios }\end{array}$ \\
\hline $\begin{array}{l}\text { San Juan del } \\
\text { Río }\end{array}$ & $\begin{array}{l}\text { Todos los usuarios jubilados, pensionados y personas } \\
\text { mayores de } 60 \text { años, que acrediten este carácter, } \\
\text { gozarán de un subsidio de hasta } 15 \mathrm{~m}^{3} \text { mensuales, de } \\
\text { los consumos registrados y sólo autorizados para uso } \\
\text { doméstico. Tiene una tarifa para la asistencia social y } \\
\text { para escuelas }\end{array}$ \\
\hline San Luis Potosí & $\begin{array}{l}\text { Hay diferenciación en cuanto a la cuota fija (popular, } \\
\text { económica y residencial) }\end{array}$ \\
\hline Tiju & $\begin{array}{l}\text { Para los pensionados, jubilados e indigentes con un } \\
\text { consumo de: 1) Hasta } 25 \mathrm{~m}^{3} \text { aplica descuento del } \\
100 \% \text {. 2) Hasta } 40 \mathrm{~m}^{3} \text {, del } 1 \text { al } 25 \text { se aplica descuento } \\
\text { del } 100 \% \text { y del } 26 \text { al } 40 \text { se aplica descuento del } 50 \% \text {. } \\
\text { 3) En consumos superiores a } 40 \mathrm{~m}^{3} \text { no hay descuento } \\
\text { alguno }\end{array}$ \\
\hline Tlas & el servicio doméstico \\
\hline Toluca & $\begin{array}{l}\text { iste diferenciación solo para tarifas de cuota fija: } \\
\text { pular rural, popular baja, popular media, popular } \\
\text { ta, residencial baja, residencial media, residencial } \\
\text { edia-alta y residencial alta }\end{array}$ \\
\hline Torreón & encontr \\
\hline \begin{tabular}{|l|} 
Tula \\
\end{tabular} & $\begin{array}{l}\text { Hay doméstica c } \\
\text { alta }\end{array}$ \\
\hline ala & \\
\hline
\end{tabular}

Fuente: resolutivos tarifarios y leyes de ingresos

Lo primero que se realizó fue categorizar cada una de las ciudades en el enfoque correspondiente. Si en la localidad, las tarifas son diferentes por más de dos extractos socioeconómicos, entonces se consideró que pertenece al enfoque de diferenciación de usuarios. Por el contrario, si la tarifa es similar para la mayor parte de los ciudadanos y sólo algunos tienen ciertos beneficios dada su comprometida situación económica, se categorizaron en el enfoque de grupos vulnerables.

Una vez categorizadas las ciudades se procedió a obtener los promedios de sus indicadores de gestión según el enfoque tarifario con la finalidad de evaluar qué perspectiva está asociada a un mejor desempeño. Es decir, se agruparon las ciudades según el criterio social utilizado (grupos vulnerables o diferenciación de usuarios) y se calcularon valores promedio de tarifa doméstica, incrementos de 2008 a 2013, promedio 
de tarifa industrial y comercial, nivel de subsidio cruzado, dotación en litros por habitante al día, dotación real, cobertura, eficiencia física, eficiencia comercial, eficiencia global y utilidad por metro cúbico.

Los promedios de los mismos indicadores mostrados en el párrafo anterior fueron contrastados entre las ciudades de "tarifa alta" y "tarifa baja". Para fines de análisis, en el presente artículo se consideran en el primer grupo a aquellas 16 ciudades con las tarifas más altas; mientras que en el segundo, las 16 más bajas.

Los análisis de promedios de indicadores, tanto en la agrupación de ciudades según el criterio social, como en la de nivel tarifario son relevantes, porque permiten contrastar y definir qué características de las tarifas de los organismos operadores de agua acompañan un mejor desempeño.

\section{Resultados}

En este apartado se observarán los resultados de la metodología planteada. En la Tabla 6 se muestra la clasificación de las ciudades según el criterio social para definir la tarifa. Resultaron ser 17 localidades las que utilizan el enfoque a grupos vulnerables, para determinar sus tarifas, y 16 las que prefieren utilizar el enfoque de diferenciación de usuarios domésticos.

Tabla 6. Enfoques para determinar tarifas desde el punto de vista social

\begin{tabular}{|c|c|c|c|}
\hline \multicolumn{2}{|c|}{ Grupos vulnerables } & \multicolumn{2}{|c|}{$\begin{array}{l}\text { Diferenciación de } \\
\text { usuarios domésticos }\end{array}$} \\
\hline $\begin{array}{l}\text { Acapulco } \\
\text { Cancún } \\
\text { Chetumal } \\
\text { Culiacán } \\
\text { Delicias } \\
\text { Ensenada } \\
\text { Guadalajara } \\
\text { Hermosillo } \\
\text { Juárez }\end{array}$ & $\begin{array}{l}\text { La paz } \\
\text { León } \\
\text { Mexicali } \\
\text { Puebla } \\
\text { San Juan del } \\
\text { Río } \\
\text { Tijuana } \\
\text { Tlaxcala } \\
\text { Torreón }\end{array}$ & $\begin{array}{l}\text { Aguascalientes } \\
\text { Atizapán } \\
\text { Campeche } \\
\text { Colima } \\
\text { Distrito Federal } \\
\text { Gómez Palacio } \\
\text { Mérida } \\
\text { Monterrey } \\
\text { Morelia }\end{array}$ & $\begin{array}{l}\text { Naucalpan } \\
\text { Oaxaca } \\
\text { San Luis Potosí } \\
\text { Toluca } \\
\text { Tula de Allende } \\
\text { Xalapa }\end{array}$ \\
\hline
\end{tabular}

Fuente: resolutivos tarifarios y páginas de internet de los organismos 
Una vez clasificadas las ciudades por enfoque tarifario, se procedió a hacer un comparativo, tanto de tarifas, como de indicadores de gestión promedio, para observar el desempeño general de los organismos bajo las dos perspectivas señaladas (diferenciación de usuarios y grupos vulnerables).

Como se puede observar en la Tabla 7, la tarifa promedio en el enfoque de diferenciación es ligeramente mayor que en el enfoque de grupos vulnerables. Esto se explica en parte porque, al haber varios tipos de usuarios es más asequible tener tarifas más altas para usuarios de altos ingresos, quienes no se ven tan afectados por una tarifa más adecuada pues en relación a sus ingresos el impacto no es tan significativo. En cambio, en el enfoque a grupos vulnerables el nivel de la tarifa es generalizado (salvo los usuarios subsidiados), lo que hace políticamente más complicado el incremento. En el mismo orden de ideas es posible observar que el incremento de 2008 a 2013, en el enfoque de diferenciación fue de $73 \%$ mientras que en el de grupos vulnerables fue $58 \%$.

En cuanto al nivel de subsidio cruzado, se aprecia que en el enfoque a grupos vulnerables es mayor que en el de diferenciación (2.91 contra 2.12). Esto sugiere que, al ser más complicado subir las tarifas domésticas en la perspectiva de grupos vulnerables, el órgano decisor de la tarifa prefiere aplicar incremento a usuarios comerciales e industriales. Como se había señalado anteriormente esta es una estrategia que reduce el impacto político, pero que afecta o desincentiva al sector empresarial y productivo.

Se observa también que el consumo real promedio es similar en ambos enfoques. Sin embargo, en cuanto a la eficiencia se puede observar que las ciudades con enfoque de grupos vulnerables tienen mejores indicadores, debido al incentivo que provoca tener una baja tarifa y, por lo tanto, menos recursos económicos para hacer frente a sus costos. Esta eficiencia tiene como resultado que las localidades con enfoque a grupos vulnerables tengan menores pérdidas financieras promedio por metro cúbico $(-2.84$ pesos contra -2.91$)$ que el enfoque de diferenciación, pese a la diferencia de tarifas. Es decir, las tarifas más bajas tienen como consecuencia que el organismo operador, bajo el enfoque a grupos vulnerables, tenga que ser más eficiente para poder suministrar el servicio a la población dado una expectativa de ingresos insuficientes para cubrir los costos operativos. 
Tabla 7. Indicadores de gestión por enfoque tarifario

\begin{tabular}{|c|c|c|}
\hline Enfoque & $\begin{array}{c}\text { E. Grupos } \\
\text { vulnerables (17) }\end{array}$ & Diferenciación (15) \\
\hline Tarifa doméstica & 9.58 & 10.68 \\
\hline $\begin{array}{lll}\text { Incremento } 2008 & - \\
2013 & & \\
\end{array}$ & $58 \%$ & $73 \%$ \\
\hline $\begin{array}{lr}\text { Promedio } & \text { tarifa } \\
\text { comercial e industrial }\end{array}$ & 24.72 & 23.27 \\
\hline $\begin{array}{l}\text { Nivel de subsidio } \\
\text { cruzado }\end{array}$ & 2.91 & 2.12 \\
\hline $\begin{array}{l}\text { Dotación } \\
\text { (Its/hab/día) }\end{array}$ & 268 & 315 \\
\hline $\begin{array}{l}\text { Dotación } \\
\text { (Its/hab/día) }\end{array}$ & 167 & 165 \\
\hline Cobertura & $97 \%$ & $94.80 \%$ \\
\hline Eficiencia física & $62.57 \%$ & $49.56 \%$ \\
\hline Eficiencia comercial & $74.09 \%$ & $71.70 \%$ \\
\hline Eficiencia global & $48.92 \%$ & $38.87 \%$ \\
\hline $\begin{array}{l}\text { Utilidad por metro } \\
\text { cúbico }\end{array}$ & -2.84 & -2.907 \\
\hline
\end{tabular}

Fuente: elaboración propia en base a CONAGUA (2014)

Lo señalado antes nos hace reflexionar acerca de la importancia que tiene que el incremento en tarifas vaya acompañado de una mayor eficiencia, tanto física como comercial. Esto ayudará a que el organismo tenga una medición adecuada de lo que se consume, para facturar lo que se mide y para cobrar lo que se factura. Ser financieramente sustentable y tener mejoras que eviten el desperdicio de agua debe ser un objetivo constante de los encargados de la administración del recurso agua en nuestro país. No es de utilidad inyectar recursos a un organismo operador, si éstos se desperdiciarán en ineficiencias y manejos inadecuados.

Una vez observados los indicadores por enfoque de determinación de tarifas se procede a analizar cómo es la gestión del agua para uso urbano según el nivel de las mismas. Tal como se puede apreciar en la Tabla 8, los municipios con tarifas más elevadas tienen, en promedio, 
significativamente menos consumo medio real de agua (151 litros por habitante al día contra 185). Y tal, como se expuso anteriormente, la pérdida por metro cúbico es similar, debido a que las localidades con baja tarifa tienen mayor nivel de subsidio cruzado y mayor eficiencia. Es decir, las ciudades con enfoque de diferenciación de usuarios tienen tarifas más altas, pero menor subsidio cruzado y menor eficiencia; mientras que las ciudades con enfoque a grupos vulnerables tienen tarifas más bajas, acompañadas de niveles de subsidio cruzado más alto y mayor eficiencia.

Tabla 8. Nivel de tarifa e indicadores de gestión

\begin{tabular}{|l|c|c|}
\hline \multicolumn{1}{|c|}{ Tarifa doméstica } & Alta & Baja \\
\hline Dotación (Its/hab/día) & 271 & 314 \\
\hline $\begin{array}{l}\text { Dotación real } \\
\text { (Its/hab/día) }\end{array}$ & 150 & 185 \\
\hline Ingresos $\left(\$ / \mathrm{m}^{3}\right)$ & 8.01 & 4.84 \\
\hline Costos $\left(\$ / \mathrm{m}^{3}\right)$ & 10.89 & 7.71 \\
\hline Utilidad $\left(\$ / \mathrm{m}^{3}\right)$ & -2.88 & -2.87 \\
\hline Nivel subsidio cruzado & 2.14 & 2.94 \\
\hline Eficiencia física & $56.86 \%$ & $56.84 \%$ \\
\hline Eficiencia comercial & $68.38 \%$ & $78.30 \%$ \\
\hline Eficiencia global & $44.12 \%$ & $44.84 \%$ \\
\hline
\end{tabular}

Fuente: elaboración propia con información de CONAGUA (2014)

\section{Conclusiones}

Con base en edl análisis planteado en las páginas anteriores, se sugiere que los modelos propuestos en este artículo tienen ventajas y desventajas. Las ventajas del enfoque a grupos vulnerables son que el subsidio, si está bien definido, se focaliza en las personas con necesidades financieras más reales; además de la existencia de menos quejas por tipificación de usuarios, debido a que, quienes no son subsidiados, tienen el mismo rango de tarifas. En lo que respecta a sus 
desventajas, destaca el mismo cobro a clientes con muy diferente poder adquisitivo; así como posibles errores de demarcación, incluyendo a personas con alto ingreso en algún grupo vulnerable.

En cuanto al enfoque de diferenciación, sus ventajas son la posibilidad de cobrar más a usuarios con un poder adquisitivo mayor; se facilita el incremento de tarifas, y requiere menos subsidio cruzado. En lo concerniente a las desventajas destacan la dificultad en identificar a los diferentes tipos de usuarios, y al facilitar una tarifa más alta, se descuida la eficiencia (Tabla 9).

Tabla 9. Ventajas y desventajas de los enfoques tarifarios

\begin{tabular}{|c|c|c|}
\hline & Grupos vulnerables & Diferenciación \\
\hline Ventajas & $\begin{array}{l}\text { - Se focaliza el } \\
\text { subsidio } \\
\text { - Menos quejas por } \\
\text { tipificación }\end{array}$ & $\begin{array}{l}\text { - Más cobro a usuarios } \\
\text { con mayor } \\
\text { adquisitivo } \\
\text { (menor } \\
\text { consumo) }\end{array}$ \\
\hline Desventajas & $\begin{array}{l}\text { - Se les cobra lo } \\
\text { mismo a clientes } \\
\text { con diferente } \\
\text { poder adquisitivo } \\
\text { - Puede existir } \\
\text { pertenencia a un } \\
\text { "grupo vulnerable" } \\
\text { teniendo alto } \\
\text { poder adquisitivo }\end{array}$ & $\begin{array}{l}\text { - Complicado identificar } \\
\text { los tipos de usuarios } \\
\text { - Al facilitar una tarifa } \\
\text { más alta hay menos } \\
\text { incentivos para la } \\
\text { eficiencia }\end{array}$ \\
\hline
\end{tabular}

Fuente: elaboración propia

Es relevante mencionar que los comentarios aquí vertidos parten de información que es reportada por los propios organismos operadores y que en ocasiones es estimada sin una verificación. Para futuros estudios sería interesante explorar si la calidad y validez de la información está acompañadas de mayores recursos económicos de los organismos, porque es posible que cuando indicadores como la eficiencia física y comercial son medidos adecuadamente, resulten valores menores que 
cuando son estimados. Asimismo, sería adecuado estudiar las características de aquellos organismos que cuenten con sistemas de verificación de información.

Existen otros factores que podrían estar asociados al nivel tarifario, como la estructura de costos, la facilidad en la recaudación a medida que las tarifas son más bajas y el factor socioeconómico asociado al consumo. Sin embargo, serán objeto de trabajos posteriores.

Aunque hay varios aspectos por explorar, según lo analizado en este documento, se puede concluir con la importancia que tiene rescatar las ventajas antes mencionadas, tanto del enfoque de diferenciación como el de grupos vulnerables; qué tarifas altas, por lo general, van acompañadas de menor consumo; $y$, lo más importante, que el incremento de tarifas debe ir acompañado de una mayor eficiencia en la gestión del agua. De nada sirven tarifas altas si los recursos no serán bien aprovechados.

\section{Referencias}

Conagua, Comisión Nacional del Agua. (2014). Situación del Subsector de Agua Potable, Alcantarillado y Saneamiento, Edición 2014. Ciudad de México, México: Secretaría de Medio Ambiente y de Recursos Naturales.

Dinar, A., Pochat, V. \& Albiac-Murillo, J. (2015). Introduction. En: Dinar, A., Pochat, V., \& Albiac-Murillo, J. (eds.). Water Pricing Experiences and Innovations (pp. 1 - 12). Switzerland: Springer.

Enouy, R., Rehan, R., Brisley, N. \& Unger, A. (2015). An Implicit Model for Water Rate Setting Within Municipal Utilities. American Water Works Association, 107, 9.

Grafton, R. Q., Chu, L. \& Kompas, T. (2015). Optimal water tariffs and supply augmentation for cost-of-service regulated water utilities. Utilities Policy, 34, 54-62.

Herrera, V. (2017). Water and Politics: Clientelism and Reform in Urban Mexico. Ann Arbor, Michigan, USA: University of Michigan Press. Recuperado

de http://www.jstor.org/stable/10.3998/mpub.9210462.

Johnson, J. A. (1971). Economic analysis of sewer service change formulas. Land Economics, 47(1), 80-86.

Molinos-Senante, M., \& Donoso, G. (2016). Water scarcity and affordability in urban water pricing: A case study of Chile. Utilities Policy, 30, 1-10. 
Nauges, C., \& Whittington, D. (2017). Evaluating the Performance of Alternative Municipal Water Tariff Designs: Quantifying the Tradeoffs between Equity, Economic Efficiency, and Cost Recovery. World Development, 91, 125-143. Recuperado de http://dx.doi.org/10.1016/j.worlddev.2016.10.014.

Pinto, S. F., \& Marques, C. R. (2015). Tariff recommendations: A Panacea for the Portuguese water sector? Utilities Policy, 34, 3644.

Rogers, P., de Silva, R., \& Bhatia, R. (2002). Water is an economic good: how to use prices to promote equity, efficiency, and sustainability. Water Policy, 4, 1-17.

Spiller, P. T., \& Savedoff, W. (1999). Commitment and governance in infrastructure sectors. En: Basañes, F., Uribe, E., \& Willig, R. (eds.). Can Privatization Deliver? Infrastructure for Latin America (pp. 133-150). Balitmore, Maryland, USA: Johns Hopkins University Press. 